\title{
Bedeutung der Herzfrequenzvariabilität in der Trainingssteuerung
}

\author{
Knechtle, Beat
}

DOI: https://doi.org/10.1024/1661-8157/a002489

Posted at the Zurich Open Repository and Archive, University of Zurich

ZORA URL: https://doi.org/10.5167/uzh-127085

Journal Article

Accepted Version

Originally published at:

Knechtle, Beat (2016). Bedeutung der Herzfrequenzvariabilität in der Trainingssteuerung. Praxis, 105(18):1051. DOI: https://doi.org/10.1024/1661-8157/a002489 


\section{Editorial}

\section{Die Bedeutung der Herzfrequenzvariabilität in der Trainingssteuerung}

Die heutigen Ausdauersportler benötigen für ihre Trainingsplanung verschiedene Hilfsmittel und Methoden. Eines dieser vielen möglichen Hilfsmittel zur Trainingssteuerung ist die Verwendung der Herzfrequenzvariabilität (HFV; engl. Heart Rate Variability, HRV). Unter der HFV versteht man die spontane Variation der Rhythmik der Herzfrequenz oder anders ausgedrückt die zeitliche Abweichung, respektive die Schwankung zwischen den einzelnen R-Zacken (R-R-Intervall).

Grundsätzlich kann man vermuten dass die Herzfrequenz eines gesunden Menschen regelmäßig ist wenn man den Puls am Handgelenk tastet. Betrachtet man die Herzfrequenz eines Herzgesunden aber genauer im EKG, so sieht man dass sie sich keineswegs regelmäßig verhält. Oft sind gewisse spontane Unregelmäßigkeiten in den zeitlichen Abständen zwischen den R-Zacken zu erkennen. Diese Unregelmäßigkeiten sind allerdings physiologisch und das Ergebnis verschiedener körpereigener physiologischer Regelmechanismen. Dieses Phänomen der Unregelmäßigkeiten ist also keinesfalls mit einer krankhaften Herzrhythmusstörung zu verwechseln, die als eine Störung der Herzfrequenz definiert wird.

Die HRV eines Herzgesunden ist im Ruhezustand am unregelmäßigsten und somit ein guter Indikator für Gesundheit und Erholung. Über autonome physiologische Regulationswege passt ein gesunder Organismus die Herzfrequenz ständig den momentanen Erfordernissen an. So haben etwa eine körperliche Beanspruchung sowie eine psychische Belastung in der Regel eine Erhöhung der Herzfrequenz zur Folge. Die erhöhte Herzfrequenz geht normalerweise bei Entlastung und Entspannung wieder zurück. Dabei zeigt sich eine höhere Anpassungsfähigkeit an Belastungen in einer größeren Variabilität der Herzfrequenz. 
Die HRV ist heute neben der Herzfrequenz die wichtigste Messgröße für eine genaue Belastungssteuerung und Leistungsdiagnostik beim Ausdauersportler. Die körperliche Verfassung eines Menschen wirkt sich stark auf die zeitlichen Abstände zwischen den RZacken aus und kann mittels EKG oder einer geeigneten Pulsuhr gemessen werden. Wird aufgrund der Messung der HRV ein körperliches Missverhältnis in Form von Stress, Übertraining oder einer Erkrankung bemerkt, ist zu empfehlen, das Training ruhiger zu gestalten oder gar auszusetzen, um die Gesundheit und den Fortlauf der Leistungssteigerung nicht zu gefährden. Vor jedem Training kann eine entsprechende Aktualisierung der Messung vorgenommen werden.

Gronwald et al. [1] haben nun in ihrer Arbeit , Evaluierung individueller Trainingsvorgaben auf Basis der Herzfrequenzvariabilität für ein Lauftraining im Freizeit- und Gesundheitssport‘ untersucht ob sich die über ein Herzfrequenzmessgerät auf der Basis der HRV-ermittelten Intensitätsbereiche in Abhängigkeit der Leistungsfähigkeit über einen mehrwöchigen Trainingszyklus verändern. Es zeigte sich über den Interventionszeitraum einer 12-wöchigen Halbmarathonvorbereitung dass sich die Unter- und Obergrenze der vorgegebenen Herzfrequenz in der niedrigsten Intensitätszone signifikant erhöhten. Bezogen auf die Ausdauerleistung waren nach der Trainingsintervention zudem signifikante Verbesserungen zu verzeichnen. Die Anpassung der Herzfrequenzwerte an die veränderte Leistungsfähigkeit sichert in diesem Rahmen ein reizwirksames Training über eine mehrwöchige Trainingsintervention ab. Für den Praktiker wichtig zu wissen ist das ein Trainingsprogramm, das die Intensitätsvorgaben von der individuellen HRV ableitet, zu einer Verbesserung der Ausdauerleistungsfähigkeit führt. 


\section{Bibliographie}

1. Gronwald T, Schulze S, Ludyga S, Hottenrott K. Evaluierung individueller Trainingsvorgaben auf Basis der Herzfrequenzvariabilität für ein Lauftraining im Freizeit- und Gesundheitssport. Praxis 2016

Prof. Dr. med. Beat Knechtle

Facharzt FMH für Allgemeinmedizin

Gesundheitszentrum St. Gallen

Vadianstrasse 26

9001 St. Gallen

Telefon $\quad+41(0) 712269300$

Telefax $\quad+41(0) 712269301$

E-Mail_beat.knechtle@hispeed.ch 